\title{
Efficient Complete Coverage Through Heterogeneous Sensing Nodes
}

\author{
Waseem Abbas, Member, IEEE, and Xenofon Koutsoukos, Senior Member, IEEE
}

\begin{abstract}
We investigate the coverage efficiency of a sensor network consisting of sensors with circular sensing footprints of different radii. The objective is to completely cover a region in an efficient manner through a controlled (or deterministic) deployment of such sensors. In particular, it is shown that when sensing nodes of two different radii are used for complete coverage, the coverage density is increased, and the sensing cost is significantly reduced as compared to the homogeneous case, in which all nodes have the same sensing radius. Configurations of heterogeneous disks of multiple radii to achieve efficient circle coverings are presented and analyzed.
\end{abstract}

Index Terms-Complete coverage, wireless sensor networks, covering density, sensing cost.

\section{INTRODUCTION}

$\mathbf{I}$ $\mathrm{N}$ the context of sensors' deployment for coverage purposes, there are two broad categories of schemes: random and deterministic. Typically, in random deployment, sensors are distributed randomly in large numbers to achieve a partial coverage (e.g., [1] and [2]). On the other hand, in deterministic schemes, complete coverage of a region can be achieved even with a limited number of sensing devices (e.g., see [3]). In deterministic deployment strategies, information regarding sensors' locations (relative positions) is required unlike random schemes. Both approaches have their own characteristics and selection of a particular approach is a matter of the application under consideration [4], [5]. However, in many real life applications in which sensing nodes are expensive and sophisticated devices, controlled (or deterministic) deployment is generally adapted and approved. For instance, in agricultural domain, to monitor climatic conditions such as temperature and humidity etc., a sensor network is deployed in which wireless sensors are spread throughout a field in the form of a grid (or other regular patterns) to completely cover a field [6]. Similarly, there are other numerous monitoring and surveillance applications in which deterministic deployment approaches are adapted for complete coverage (e.g., [1] and [4]).

In this paper, we examine the coverage efficiency of deterministic deployments of sensing nodes with circular footprints of two different radii. It is shown that for complete coverage purposes, configurations of heterogeneous disks, i.e., disks with different radii, are indeed better as compared to the homoge-

Manuscript received July 29, 2014; revised August 26, 2014; accepted September 10, 2014. Date of publication September 17, 2014; date of current version February 18, 2015. This work was supported in part by the National Science Foundation under Grants CNS-1238959 and CNS-1035655 and in part by the Air Force Research Laboratory under Award FA8750-14-2-0180. The associate editor coordinating the review of this paper and approving it for publication was C. Cordeiro.

The authors are with the Institute for Software Integrated Systems, Vanderbilt University, Nashville, TN 37212 USA (e-mail: waseem.abbas@ vanderbilt.edu; xenofon.koutsoukos@vanderbilt.edu).

Color versions of one or more of the figures in this paper are available online at http://ieeexplore.ieee.org.

Digital Object Identifier 10.1109/LWC.2014.2358631

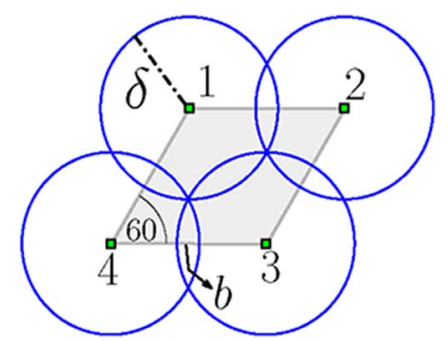

Fig. 1. Four disks of equal radii are taking part in covering a crystallographic unit of area $\frac{\sqrt{3}}{2} b^{2}$. The areas of circular discs $1,2,3$, and 4 covering a portion of crystallographic unit are $\frac{\pi \delta^{2}}{3}, \frac{\pi \delta^{2}}{6}, \frac{\pi \delta^{2}}{3}$, and $\frac{\pi \delta^{2}}{6}$, respectively. The covering density is $\rho=\frac{\sqrt{3} b^{2}}{2 \pi \delta^{2}}$.

neous case. We propose two different configurations of heterogeneous disks and analyze variations in covering densities (a metric to quantify the efficiency of covering) with respect to variations in disks radii. Relationships among disks radii to ensure complete coverage are also computed. From energy consumption perspectives in sensor networks, improvements offered by heterogeneous configurations are also demonstrated using a sensing cost metric.

Coverage problems, specially deterministic ones, find their roots in computational geometry (e.g., [7] and [8]). One of the primary problem is related to circle covering; what is the most efficient way to place circular disks of same radii to completely cover a region. The solution is provided in [7] stating that the most efficient way minimizing overlaps among disks is to place disks in an equilateral triangle lattice. In [9], a lower bound on the density of covering is given when circular disks of two different radii are used. In multiagent and sensor networks, circular disks correspond to the sensing footprints of nodes and minimizing overlap means that the power is consumed efficiently for the sensing operation.

\section{EFFICIENCY OF COVERAGE}

In this section, we provide a formal description of the efficiency of coverage in terms of the coverage density and sensing cost. A circle covering is a configuration of overlapping circles with given radii to completely cover some domain in $\mathbb{R}^{d}$ ( $d=2$ in our case). In order to quantify the efficiency of circle covering, a commonly used metric is the covering density, denoted by $\rho$. It measures how efficiently circular disks are arranged to cover the given domain. The notion of crystallographic unit [10] of the circle covering is needed to define $\rho$. Crystallographic unit of a covering is a parallelogram containing the minimum repeatable elements of a circle covering, as shown in Fig. 1.

1) Definition 2.1: Covering Density: The covering density of a circle covering, denoted by $\rho$, is the ratio of the area of the crystallographic unit to the area of circular disks covering the crystallographic unit. 
Let $c$ be a crystallographic unit of a circle covering and $A(c)$ be the area of $c$, then

$$
\rho=\frac{A(c)}{\sum_{j=1}^{k} x_{j}\left(\pi \delta_{j}^{2}\right)} .
$$

Here, $k$ is the number of circular disks that are covering $c, x_{j}$ is the fraction of the area of the circular disk of radius $\delta_{j}$ covering a portion of $c$. An example is shown Fig. 1.

A circle covering with a higher $\rho$ is more efficient. It means that the overlap among circular disks is smaller and the areas of disks are being utilized efficiently to cover the region. In practical scenarios, these circular disks may correspond to sensing footprints of the sensors deployed within some region. Overall energy consumed by sensors for sensing operations is directly dependent on the area of footprints.

In fact, in a sensor range model based on RF-power density function for an isotropic antenna, power consumed is directly proportional to the area of the sensor footprint given by $\pi\left(\delta_{j}\right)^{2}$, where $\delta_{j}$ is the radius of the footprint (e.g., [11]). Thus, a sensing cost (in terms of the power consumption) can be associated with a sensor that is proportional to the square of the radius of the footprint of the sensor. To efficiently utilize energy resources, sensors should be deployed to maximally utilize the area of circular footprints. Therefore, a sensing cost can be associated with a configuration of a group of nodes covering a region.

2) Definition 2.2: Sensing Cost: Let there be $n$ agents with sensing footprints of circular shapes, and $\delta_{j}$ be the radius of the footprint of agent $j \in\{1,2, \cdots, n\}$, then the overall sensing cost of the system is

$$
\mathcal{J}=\sum_{j=1}^{n}\left(\delta_{j}\right)^{2} .
$$

Like the density, $\mathcal{J}$ is also a measure of the efficiency of the circle covering from the energy consumption perspective. If $x$ and $y$ are two configurations with exactly same number of nodes and covering the same region such that $\mathcal{J}_{y}<\mathcal{J}_{x}$, then $y$ is indeed a better choice from the sensing cost view-point. A large amount of overlap among circles in a circle covering results into an increase in the sensing cost. Covering density and sensing cost will both be used to compute the efficiency of circle coverings in subsequent sections.

\section{Node Configurations for Circle Covering}

In this section, we present two different circle coverings (heterogeneous configurations), each of which employ circular discs of two different radii. But first, details of the optimal circle covering through circular disks of same radii (homogeneous configuration) are stated.

\section{A. Homogeneous Configuration}

Let there be a group of agents each having a sensing footprint of radius $\delta$. It is shown in [7] that for the complete covering purpose, the optimal way is to place agents on an equilateral triangle lattice as shown in Fig. 2. The lines connecting nodes, whose sensing footprints intersect, form an equilateral triangle with the length of each side being equal to $\sqrt{3} \delta$. Moreover,

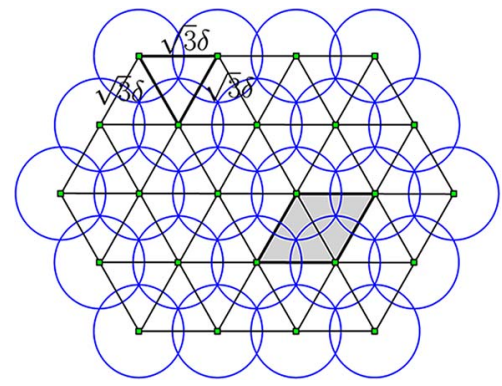

Fig. 2. Circle covering through circular disks of same radii. The shaded region is a crystallographic unit.

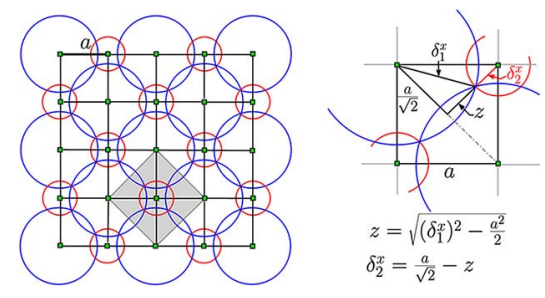

Fig. 3. Heterogeneous configuration 1 in which the shaded parallelogram is a crystallographic unit of the covering. For a given $\delta_{1}^{x}$, appropriate value of $\delta_{2}^{x}$ is also computed

the density of the covering, denoted by $\rho_{h}$, is $\frac{\sqrt{27}}{2 \pi}$ which is the best possible. ${ }^{1}$ If there are $n$ agents, the sensing cost of the homogeneous configuration is $\mathcal{J}_{h}=n \delta^{2}$.

\section{B. Heterogeneous Configurations}

Using heterogeneous disks (in terms of the radii) for the purpose of circle covering may further improve the efficiency of covering. We present two heterogeneous configurations, denoted by $X$ and $Y$, respectively. It is shown in the next section that they are indeed better than the homogeneous case from the covering density and the sensing cost perspectives.

1) Configuration $1(X)$ : Let there be two types of nodes with respect to the radii of sensing footprints, which are denoted by $\delta_{1}^{x}$ and $\delta_{2}^{x}$, respectively. The superscript in the notation indicates the configuration and the subscript corresponds to the node type. Moreover, assume that $\delta_{2}^{x}<\delta_{1}^{x}$. These two types of nodes are placed on a grid network alternatively as shown in Fig. 3. If the length of the side of each square in the grid is $a$, then the $X$ configuration results into a circle covering of the region if $\delta_{1}^{x}$ and $\delta_{2}^{x}$ satisfy the following conditions:

$$
\begin{aligned}
& \delta_{1}^{x}=\epsilon_{x} a, \quad \frac{1}{\sqrt{2}} \leq \epsilon_{x} \leq 1 \\
& \delta_{2}^{x}=\frac{1}{\sqrt{2}}\left(a-\sqrt{\left(2 \delta_{1}^{x}\right)^{2}-a^{2}}\right) .
\end{aligned}
$$

A crystallographic unit of the circle covering as a result of $X$ is shown in Fig. 3. Thus, the density of the covering, denoted by $\rho_{x}$, is

$$
\rho_{x}=\frac{(\text { crystallographic unit area) }}{\pi\left[\left(\delta_{1}^{x}\right)^{2}+\left(\delta_{2}^{x}\right)^{2}\right]}=\frac{2 a^{2}}{\pi\left[\left(\delta_{1}^{x}\right)^{2}+\left(\delta_{2}^{x}\right)^{2}\right]} .
$$

\footnotetext{
${ }^{1}$ It is assumed that the area being monitored is much larger than the sensing footprint of an individual node.
} 


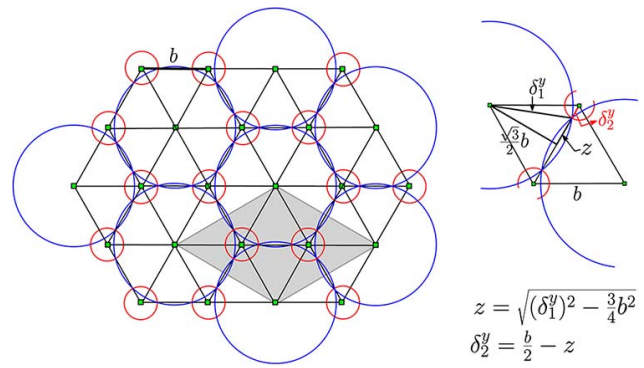

Fig. 4. Heterogeneous configuration 2. A crystallographic unit is a shaded parallelogram. A relationship between $\delta_{1}^{y}$ and $\delta_{2}^{y}$ to obtain a circle covering is also shown.

2) Configuration $2(Y)$ : As in the case of $X$, we consider two types of nodes; one with the sensing footprints of radius $\delta_{1}^{y}$, and the other having radius $\delta_{2}^{y}$, where $\delta_{2}^{y}<\delta_{1}^{y}$. Again, the superscript indicates the configuration type and the subscript represents the node type. These nodes are placed on an equilateral triangle lattice as shown in Fig. 4 . Let $b$ be the length of the side of the unit equilateral triangle in the network, then $\delta_{1}^{y}$ and $\delta_{2}^{y}$ need to satisfy the following conditions to obtain a circle covering from this configuration of nodes as illustrated in Fig. 4.

$$
\begin{aligned}
& \delta_{1}^{y}=\epsilon_{y} b, \quad \frac{\sqrt{3}}{2} \leq \epsilon_{y} \leq 1 ; \\
& \delta_{2}^{y}=\frac{1}{2}\left[b-\sqrt{4\left(\delta_{1}^{y}\right)^{2}-3 b^{2}}\right] .
\end{aligned}
$$

The density of $Y$, denoted by $\rho_{y}$, can be obtained by computing the area of the crystallographic unit of the covering (as shown in Fig. 4). It should be noted that the area of circles, which cover a crystallographic unit, is equal to the sum of the areas of a single circle with radius $\delta_{1}^{y}$, and two circles with radius $\delta_{2}^{y}$. Thus,

$\rho_{y}=\frac{\text { (crystallographic unit area) }}{\pi\left[\left(\delta_{1}^{y}\right)^{2}+2\left(\delta_{2}^{y}\right)^{2}\right]}=\frac{\frac{3 \sqrt{3}}{2} b^{2}}{\pi\left[\left(\delta_{1}^{y}\right)^{2}+2\left(\delta_{2}^{y}\right)^{2}\right]}$.

\section{Comparison of Homogeneous And Heterogeneous Configurations}

In this section, we compute and compare coverage densities and sensing costs of configurations introduced in Section III, thereby establishing that heterogeneous configurations can outperform homogeneous configuration.

\section{A. Comparison of Coverage Densities}

The maximum coverage density for homogeneous case is $\rho_{h}=\frac{\sqrt{27}}{2 \pi}$. The covering densities for heterogeneous configurations 1 and 2 are given in (3) and (4), respectively. It is to be noted that $\rho_{i}$ depends on the values of $\delta_{1}^{i}$ and $\delta_{2}^{i}$ for $i \in\{x, y\}$. If radii ratio of disks is defined as $\alpha_{i}=\delta_{2}^{i} / \delta_{1}^{i}$ for $i \in\{x, y\}$, then the covering density directly depends on $\alpha_{i}$. Note that the radii ratio is 1 in the homogeneous case.

If covering densities of homogeneous as well as heterogeneous configurations are plotted with respect to the radii ratio,

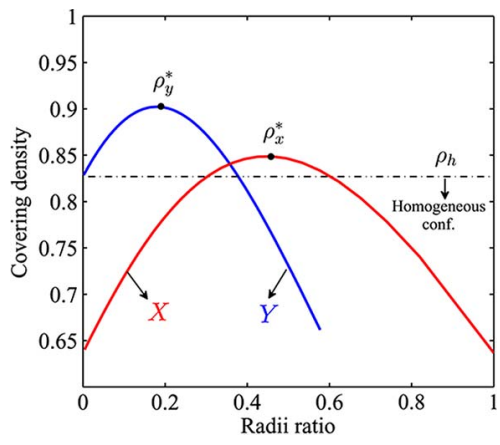

Fig. 5. Plots of covering densities of different configurations as a function of radii ratio.

the plot shown in Fig. 5 is obtained. It is observed that for quite a good range of the radii ratio, heterogeneous configurations have better covering densities than the homogeneous case, specially when the radii ratios are smaller. For the heterogeneous configuration 1 , the maximum covering density, denoted by $\rho_{x}^{*}$, is $\left(\frac{8}{3 \pi}\right)$ with $\alpha_{x}=\frac{1}{\sqrt{5}}$. Similarly, for the heterogeneous configuration 2 , the maximum covering density, denoted by $\rho_{y}^{*}$, is $\frac{18 \sqrt{3}}{11 \pi}$ which is obtained with $\alpha_{y}=\frac{1}{\sqrt{31}}$. It is to be noted that

$$
\rho_{h}<\rho_{x}^{*}<\rho_{y}^{*}
$$

Thus, heterogeneous configurations $X$ and $Y$ have improved coverage densities as compared to the homogeneous case.

\section{B. Comparison of Sensing Costs}

Next, we compare homogeneous configuration with heterogeneous configurations using sensing cost metric defined in (2). In homogeneous configuration, nodes are placed in an equilateral triangle network. Let $\tau$ be an $m \times n$ equilateral triangle network in which $b$ is the distance between two adjacent nodes. ${ }^{2}$ The area covered by the network is

$$
A_{\tau}=2(n-1)(m-1) \frac{\sqrt{3}}{4} b^{2} .
$$

In $X$, nodes are placed in a grid network. Consider an $m \times$ $n$ square grid network in which the distance between any two adjacent nodes is $a$, then the area of the grid is

$$
A_{g}=(n-1)(m-1) a^{2} .
$$

Since a comparison of sensing costs of homogeneous configuration and $X$ is desired, a grid network with the same number of nodes and area as the triangular network $\tau$ is needed. Thus, for an equilateral triangle network with a given $b$, an equivalent grid network with $a$ is obtained by equating (6) and (7) as

$$
a=(3 / 4)^{\frac{1}{4}} b .
$$

Next, sensing cost of the homogeneous configuration, in which nodes with sensing footprints of radius $\delta=\frac{b}{\sqrt{3}}$ are placed on the vertices of an $m \times n$ triangle network $\tau$ to obtain

\footnotetext{
${ }^{2}$ Nodes are arranged in an equilateral triangle network such that there are $m$ rows of nodes with $n$ nodes in each row.
} 
a circle covering of an area $A_{\tau}$, is computed. The sensing cost, denoted by $\mathcal{J}_{h}$, is

$$
\mathcal{J}_{h}=\sum_{i=1}^{m n}\left(\frac{b}{\sqrt{3}}\right)^{2}=\frac{m n b^{2}}{3} .
$$

Afterwards, nodes with sensing footprints of radii $\delta_{1}^{x}=\epsilon_{1} a$ and $\delta_{2}^{x}=\frac{1}{\sqrt{2}}\left(a-\sqrt{2\left(\delta_{1}^{x}\right)^{2}-a^{2}}\right)$, where $\epsilon_{1} \in\left[\frac{1}{\sqrt{2}} 1\right]$ and $a$ is given by (8), are placed in $X$ to obtain a circle covering of area $A_{g}\left(=A_{\tau}\right)$. The sensing cost of such a configuration is

$$
\begin{aligned}
\mathcal{J}_{x}\left(\epsilon_{x}\right) & =\frac{m n}{2}\left[\left(\delta_{1}^{x}\right)^{2}+\left(\delta_{2}^{x}\right)^{2}\right]=\frac{m n}{2} a^{2}\left[2 \epsilon_{x}^{2}-\sqrt{2 \epsilon_{x}^{2}-1}\right] \\
& =\frac{\sqrt{3}}{4} m n b^{2}\left[2 \epsilon_{x}^{2}-\sqrt{2 \epsilon_{x}^{2}-1}\right] .
\end{aligned}
$$

In fact, $\mathcal{J}_{x}\left(\epsilon_{x}\right)<\mathcal{J}_{h}$ when $0.751 \leq \epsilon_{x} \leq 0.84$. The minimum $\mathcal{J}_{x}$ is obtained for $\epsilon_{x}^{*}=\sqrt{\frac{5}{8}}$. Moreover, for $\delta_{1}^{x}=\epsilon_{x}^{*} a$, we get $\alpha_{x}=\frac{1}{\sqrt{5}}$ which is the radii ratio that gives the maximum covering density for $X$. Computing the ratio of $\mathcal{J}_{x}\left(\epsilon_{x}^{*}\right)$ to $\mathcal{J}_{h}$ gives

$$
\frac{\mathcal{J}_{x}\left(\epsilon_{x}^{*}\right)}{\mathcal{J}_{h}}=\frac{9 \sqrt{3}}{16}<1
$$

Thus, $X$ can be more economical than the homogeneous configuration from a sensing cost perspective.

Similarly, a comparison between homogeneous and heterogeneous configuration $2(Y)$ can be performed by placing nodes with sensing footprints of radii $\delta_{1}^{y}=\epsilon_{2} b$ and $\delta_{2}^{y}=$ $\frac{1}{2}\left[b-\sqrt{4\left(\delta_{1}^{y}\right)^{2}-3 b^{2}}\right]$ on the vertices of an $m \times n$ equilateral triangle network to get a circle covering. Here, $\epsilon_{y} \in\left[\frac{\sqrt{3}}{2} 1\right]$. The sensing cost of this configuration of nodes is ${ }^{3}$

$$
\begin{aligned}
\mathcal{J}_{y}\left(\epsilon_{y}\right) & =\frac{m n}{3}\left[\left(\delta_{1}^{y}\right)^{2}+2\left(\delta_{2}^{y}\right)^{2}\right] \\
& =\frac{m n b^{2}}{3}\left[3 \epsilon_{y}^{2}-1-\sqrt{4 \epsilon_{y}^{2}-3}\right] .
\end{aligned}
$$

For $0.882 \leq \epsilon_{y}<1, \mathcal{J}_{y}\left(\epsilon_{y}\right)<\mathcal{J}_{h}$. In fact, $\mathcal{J}_{y}$ is minimum at $\epsilon_{y}^{*}=\sqrt{\frac{31}{36}}$. The maximum covering density for $Y$ is also achieved when $\delta_{1}^{y}=\epsilon_{y}^{*} b$. The ratio of $\mathcal{J}_{y}\left(\epsilon_{y}^{*}\right)$ to $\mathcal{J}_{h}$ is

$$
\frac{\mathcal{J}_{y}\left(\epsilon_{y}^{*}\right)}{\mathcal{J}_{h}}=\frac{11}{12}<1 \text {. }
$$

Thus, $Y$ is more efficient than the homogeneous covering if an appropriate value of $\epsilon_{y}$ is chosen.

1) Proposition 4.1: It is possible to have a lower sensing cost and a higher covering efficiency for circle coverings obtained through heterogeneous configurations 1 and 2 as compared to the homogeneous configuration. In fact,

$$
\begin{aligned}
& \mathcal{J}_{x}\left(\epsilon_{x}\right)<\mathcal{J}_{h}, \quad \rho_{x}>\rho_{h}, \quad \text { for } \quad 0.751 \leq \epsilon_{x} \leq 0.84 \text {; } \\
& \mathcal{J}_{y}\left(\epsilon_{y}\right)<\mathcal{J}_{h}, \quad \rho_{y}>\rho_{h}, \quad \text { for } \quad 0.882 \leq \epsilon_{y} \leq 1 .
\end{aligned}
$$

\footnotetext{
${ }^{3}$ In heterogeneous configuration 2 , there are twice as many circles with $\delta_{2}$ radius as compared to circles with $\delta_{1}$ radius as long as both $\pi\left(\delta_{1}\right)^{2}$ and $\pi\left(\delta_{2}\right)^{2}$ are significantly smaller than $A_{g}$.
}

Moreover,

$$
\mathcal{J}_{y}\left(\epsilon_{y}^{*}\right)<\mathcal{J}_{x}\left(\epsilon_{x}^{*}\right)<\mathcal{J}_{h},
$$

where $\epsilon_{x}^{*}=\sqrt{\frac{5}{8}}$ and $\epsilon_{y}^{*}=\sqrt{\frac{31}{36}}$.

\section{Conclusion}

In this paper, we examined the complete coverage problem when circular disks of two different radii are used. It is shown that more efficient coverage can be obtained through configurations of heterogeneous disks as compared to the homogeneous case. In the case of heterogeneous configuration 1, best results are obtained when sensing radius of one type of nodes is approximately half $\left(\frac{1}{\sqrt{5}}\right)$ of the others, whereas in heterogeneous configuration 2 , optimum coverage density and sensing cost are obtained when the radius of one disk is approximately one-fifth $\left(\frac{1}{\sqrt{31}}\right)$ of the other. In sensor networks, to efficiently utilize energy resources, these results can be useful to control the power levels of sensing nodes which are deployed to completely cover some region.

Depending on the application, heterogeneous configurations might incur higher initial deployment costs owing to the sensors with different sensing ranges or footprints. However, reduced operational costs of heterogeneous configurations might compensate for their higher initial deployment cost. In future, we aim to analyze and compare the efficiency of various heterogeneous configurations using a more generalized sensing cost model than (2), and construct circle coverings with the maximum covering densities (or lowest sensing costs) for any given radii ratio. The effect of noise or sensor failure on the coverage property of heterogeneous configurations is another interesting direction in the context of this work.

\section{REFERENCES}

[1] I. Akyildiz and M. Vuran, Wireless Sensor Networks. New York, NY, USA: Wiley, 2010.

[2] M. Cardei and J. Wu, "Energy-efficient coverage problems in wireless adhoc sensor networks," Comput. Commun., vol. 29, no. 4, pp. 413-420, Feb. 2006.

[3] H. Zhang and J. C. Hou, "Is deterministic deployment worse than random deployment for wireless sensor networks," in Proc. IEEE INFOCOM, 2006, pp. 1-13.

[4] F. Wang and J. Liu, "Networked wireless sensor data collection: Issues, challenges, approaches," IEEE Commun. Surveys Tuts., vol. 13, no. 4, pp. 673-687, 2011

[5] H. Zhang and J. Hou, "Maintaining sensing coverage and connectivity in large sensor networks," Ad Hoc Sens. Wireless Netw., vol. 1, pp. 89-124, 2005.

[6] H. Sahota, R. Kumar, and A. Kamal, "A wireless sensor network for precision agriculture and its performance," Wireless Commun. Mobile Comput., vol. 11, no. 12, pp. 1628-1645, Dec. 2011.

[7] R. Kershner, "The number of circles covering a set," Am. J. Math., vol. 61, no. 3, pp. 665-671, Jul. 1939.

[8] G. F. Tóth and W. Kuperberg, "A survey of recent results in the theory of packing and covering," in New Trends in Discrete and Computational Geometry. Berlin, Germany: Springer-Verlag, 1993, pp. 251-279.

[9] F. Tóth, "Covering the plane with two kinds of circles," Discrete Computational Geometry, vol. 13, no. 1, pp. 445-447, 1995.

[10] R. Williams, The Geometrical Foundation of Natural Structure: A Source Book of Design. New York, NY, USA: Dover, 1979.

[11] H. Jaleel, A. Rahmani, and M. Egerstedt, "Probabilistic lifetime maximization of sensor networks," IEEE Trans. Autom. Control, vol. 58, no. 2, pp. 534-539, Feb. 2013. 